\title{
The L 1157 protostellar outflow imaged with the Submillimeter Array (Corrigendum)
}

\author{
A. I. Gómez-Ruiz ${ }^{1,2, \star}$, N. Hirano ${ }^{3}$, S. Leurini ${ }^{2}$, and S.-Y. Liu ${ }^{3}$ \\ 1 INAF, Osservatorio Astrofisico di Arcetri, Largo E. Fermi 5, 50125 Firenze, Italy \\ e-mail: aigomez@inaoep.mx \\ 2 Max-Planck-Institut für Radioastronomie (MPIfR), Auf dem Hügel 69, 53121 Bonn, Germany \\ 3 Academia Sinica, Institute of Astronomy \& Astrophysics, PO Box 23-141, Taipei, 106, Taiwan
}

A\&A 558, A94 (2013), DOI: 10.1051/0004-6361/201118473

Key words. Shock waves - ISM: jets and outflows - Stars: formation - ISM: individual objects: L1157 - errata, addenda

An error occurred during the production process. The text of Sect. 4.1.1 was missing. This whole section is displayed here.

\subsubsection{SiO high-velocity clumps}

Using the line ratio between the $\mathrm{SiO}(5-4)$ and the $\mathrm{SiO}(2-1)$ observed by Zhang et al. (2000) with a synthesized beam of 9 .' $5 \times 8$.' 0 , we derived the physical conditions of the gas in the high-velocity clumps. For our analysis we selected the portion of the high-velocity range that is less affected by the missing flux. The analysis was performed at the B0i and B1a positions, at which the $\mathrm{SiO}(5-4)$ emission was detected above the $5 \sigma$ level. We note that an analysis using several SiO lines observed with a single-dish telescope has been made by Nisini et al. (2007). However, they used single-pointing observations, which means that the data were taken with different beam sizes, for instance $\sim 27^{\prime \prime}$ for $\mathrm{SiO}(2-1)$ and $\sim 11^{\prime \prime}$ for $\mathrm{SiO}(5-4)$. In addition, the line ratios used the total integrated intensity, including the low-velocity component, where as we used higher-resolution data and focused on the high-velocity component.

Since the interferometric $\mathrm{SiO}(2-1)$ observations agreed to better than $20 \%$ with the single-dish results, Zhang et al. (2000) concluded that most of the $\mathrm{SiO}(2-1)$ flux was recovered with the interferometer. Therefore we did not take into account the effect of the missing flux in this transition. Our $\mathrm{SiO}$ (5-4) observations were convolved to the angular resolution of the $\mathrm{SiO}$ (2-1) data (i.e. 9!'5×8'.0). In Fig. 10 we show the $\mathrm{SiO}(5-4)$ and (2-1) spectra at the position of clumps B0i and B1a in a brightness-temperature scale. The portion of the high-velocity range in which most of the single-dish $\mathrm{SiO}(5-4)$ emission at the $\mathrm{B} 1 \mathrm{a}$ position is recovered by our SMA observations is -16.0 to $-10.8 \mathrm{~km} \mathrm{~s}^{-1}$. We assumed the velocity range for the B0i clump to be the same as that of the B1a clump. The linewidth was assumed to be $5 \mathrm{~km} \mathrm{~s}^{-1}$ for both B1a and B0i. Since smoothing the SMA maps to $\sim 9^{\prime \prime}$ beam averaged the emission of adjacent clumps, the spectrum at B1a includes the emission from

\footnotetext{
^ Member of the International Max-Planck Research School for Astronomy and Astrophysics at the Universities of Bonn and Cologne.
}

B1g, B1h, B1c, and B1f. However, the emission from adjacent clumps appears only in the low-velocity range and does not affect the analysis. The integrated line intensities of $\mathrm{SiO}(2-1)$ and (5-4) in this velocity range are $1.5 \pm 0.6$ and $1.5 \pm 0.1 \mathrm{~K} \mathrm{~km} \mathrm{~s}^{-1}$ at B0i, and $7.7 \pm 0.6$ and $4.4 \pm 0.1 \mathrm{~K} \mathrm{~km} \mathrm{~s}^{-1}$ at B1a. The derived $(2-1) /(5-4)$ ratios are therefore $1.0 \pm 0.4$ for $\mathrm{B} 0 \mathrm{i}$ and $1.7 \pm 0.1$ for B1a.

We used the non-local thermodynamic equilibrium program RADEX (Van der Tak et al. 2007) in the large velocity gradient (LVG) approximation and plane-parallel geometry to model the $(2-1) /(5-4)$ ratios. Using the RADEX offline distribution ${ }^{1}$, we estimated the kinetic temperature $\left(T_{\text {kin }}\right)$ and/or the volume density $(n)$ from the observed line ratio. The input parameters were the background radiation field (the $\mathrm{CMB}$ temperature of $2.73 \mathrm{~K}$ ) and the line width $\left(5 \mathrm{~km} \mathrm{~s}^{-1}\right)$. To constrain the $\mathrm{SiO}$ column density, $N(\mathrm{SiO})$, we used the observed integrated line intensity of the $\mathrm{SiO}$ (5-4) emission (1.5 and $4.4 \mathrm{~K} \mathrm{~km} \mathrm{~s}^{-1}$ for B0i and B1a, respectively). Then, by running the LVG for different $N(\mathrm{SiO})$, we found the best $N(\mathrm{SiO})$ that matches better the $\mathrm{SiO}(5-4)$ brightness temperature and the $(2-1) /(5-4)$ ratio.

In Fig. 11 we show the LVG results for the two cases of interest. The LVG results show that the $(2-1) /(5-4)$ ratio depends on the density and is less sensitive to the temperature if the density is lower than $10^{6.5} \mathrm{~cm}^{-3}$. On the other hand, the $(2-1) /(5-4)$ ratio becomes sensitive to the temperature if the density is higher than $10^{6.5} \mathrm{~cm}^{-3}$. We found that the observed ratios yield similar solutions for the two positions, with a density of $n \sim 10^{5}$ to $10^{6} \mathrm{~cm}^{-3}$. Although the uncertainties on the line intensity and ratio are larger at B0i, these uncertainties do not affect the solution significantly (less than a factor of three). It should be noted that the $N(\mathrm{SiO})$ at $\mathrm{B} 1 \mathrm{a}$ is twice as high as that at B0i. The density obtained here is similar to the $3 \times 10^{5} \mathrm{~cm}^{-3}$ derived by Nisini et al. (2007) as an averaged density that included the low-velocity component. Nisini et al. also found that the physical parameters of the high-velocity component are different from those averaged over all the emitting gas; the $(8-7) /(5-4)$ ratio at $V_{\mathrm{LSR}} \sim-15 \mathrm{~km} \mathrm{~s}^{-1}$ required either a

1 http://www.sron.rug.nl/ vdtak/radex/ 
higher density of $\sim 5 \times 10^{6} \mathrm{~cm}^{-3}$ or a higher temperature of $T_{K}>500 \mathrm{~K}$. Although our results do not support the high density of $>10^{6.5} \mathrm{~cm}^{-3}$, they do not exclude the high-temperature solution. The kinetic temperatures derived from other warm gas tracers such as high- $J$ transitions of $\mathrm{CO}, \mathrm{H}_{2}$, and $\mathrm{H}_{2} \mathrm{O}$ also support the presence of a warm gas component with $\sim 500 \mathrm{~K}$ (e.g. Nisini et al. 2007, 2010). We note that the density and temperature of the high-velocity clump are similar to those of the EHV bullets in highly collimated outflows such as HH211 and L1448C, $n \sim 10^{5}-10^{6} \mathrm{~cm}^{-3}$, and $T_{K} \geq 300 \mathrm{~K}$ (Nisini et al. 2002; Nisini et al. 2007; Hirano et al. 2006; Palau et al. 2006). This implies that the high-velocity emission in the B1a and B0i clumps has an origin common with that of the EHV bullets. 